causal effect of early age maturation on overweight. Also, there is no explanation for the gender effect.

\section{P2-56 PREVALENCE, AWARENESS, AND CONTROL OF HYPERTENSION AMONG POPULATION AGED OVER 40 YEARS IN MONGOLIA: A BASELINE SURVEY OF MONCOHORT STUDY}

doi:10.1136/jech.2011.142976h.91

${ }^{1} \mathrm{D}$ Davaakham, ${ }^{1,2} \mathrm{~T}$ Enkhoyun, ${ }^{2} \mathrm{Y}$ Nakamura. ${ }^{1}$ Department of Epidemiology and Biostatistics, Health Sciences University of Mongolia, Ulaanbaatar, Mongolia; ${ }^{2}$ Department of Public Health, Jichi Medical University, Shimotsuke, Japan

Introduction Hypertension is an important public health problem in Mongolia with a rapidly increasing incidence and deaths noted during the last several years. This study estimates the prevalence and control of hypertension among rural and urban population in Mongolia.

Methods A nationwide cross-sectional study was conducted using stratified multistage sampling. Two blood pressure measurements were obtained using a standardised mercury sphygmomanometer. Information on history of hypertension was obtained using a standard questionnaire. Hypertension was defined as mean systolic and diastolic BPs at $>140 \mathrm{~mm} \mathrm{Hg}$ and $>90 \mathrm{~mm} \mathrm{Hg}$, and/or self-reported current use of antihypertensive medication, previous diagnosis.

Results A total of 2280 people aged over 40 years were examined (response rate $93.5 \%$ ). Overall, $63.8 \%$ of study population had hypertension. Around 11\% were newly diagnosed whereas $55.4 \%$ were aware of their hypertension. Prevalence of hypertension significantly increased with age $(p=0.0001)$. In addition women were more likely to be aware of their disease and to be on treatment compared to men. People who are aware of their hypertension were significantly more likely to quit smoking compared to those who are newly diagnosed and who are non-hypertensive $(22.0 \%, 6.7 \%$ and $11.8 \%, \mathrm{p})$. In addition they were more likely to reduce consumption of alcohol (alcohol consumption $56.7 \%, 65.1 \%$ and $64.6 \%, p=0.001$ ) to be on diet $(29.2 \%, 5.3 \%$ and $2.6 \%, \mathrm{p}=0.0001)$, and reducing their weights $(17.1 \%, 5.3 \%$ and $0 \%, \mathrm{p}=0.0001)$.

Conclusions Hypertension is highly prevalent among population aged over 40 years. Hypertensive people who are aware of their disease were more likely to reduce their risk behaviours and to promote healthy lifestyles.

\section{P2-57 DIABETES, OBESITY AND SOME RISK FACTORS AMONG ADULTS IN MONGOLIA: A NATIONWIDE CROSS-SECTIONAL SURVEY}

doi:10.1136/jech.2011.142976h.92

${ }^{1} \mathrm{D}$ Davaalkham, ${ }^{1,2} \mathrm{~T}$ Enkhoyun, ${ }^{*}{ }^{2} Y$ Nakamura. ${ }^{1}$ Department of Epidemiology and Biostatistics, Health Sciences University of Mongolia, Ulaanbaatar, Mongolia; ${ }^{2}$ Department of Public Health, Jichi Medical University, Shimotsuke, Japan

Introduction To determine the population-based prevalence of diabetes, impaired fasting glucose (IFG), obesity and non-communicable disease risk factors in Mongolia.

Methods A national sample involving 2280 participants aged $\geq 40$ years were examined in a cross-sectional survey conducted in 2009. The WHO diagnostic criteria were used to determine the prevalence of diabetes, IFG and obesity. Serum samples were tested for cholesterol, triglyceride and lipoproteins.

Results Around $55 \%$ of the participants were enrolled from capitalUlaanbaatar city, $30.1 \%$ were from rural soums of different geographical regions and the remaining was residing in the province centers (response rate was $93.5 \%$ ). The diabetes prevalence was $8.0 \%$, and an additional $7.4 \%$ had IFG. Prevalence of diabetes in the city, province centers and rural soums was $9.8 \%, 6.4 \%$ and $5.5 \%$ with the significantly highest rate in the city $(p=0.012)$. The prevalence of diabetes among population aged over 40 years was $8.3 \%$ in men and $7.8 \%$ in women. The prevalence of overweight, obesity and central obesity was $37.4 \%, 26.9 \%$ (overall $64.3 \%$ ) and $63.6 \%$, respectively. Among study population the rates of abnormal triglyceride, cholesterol, and high and low density lipoprotein rates were $14.1 \%, 16.2 \%, 28.2 \%$, and $14.0 \%$, respectively. Overall smoking and alcohol consumption rates were $24 \%$ and $60.3 \%$, with significant higher use among men compared to women. In general, diet, smoking, alcohol consumption and physical activity were different by age and residence.

Conclusions Mongolia has a rapidly rising prevalence of diabetes and obesity. Central obesity is strongly correlated with adverse health outcomes. In addition unhealthy behaviours are emerging in the adult population.

\section{P2-58 UNHEALTHY LIFESTYLE PATTERNS ASSOCIATED WITH WAIST CIRCUMFERENCE AMONG ADOLESCENTS: A SCHOOL BASED SURVEY}

doi:10.1136/jech.2011.142976h.93

${ }^{1,2} \mathrm{~A}$ C de Moraes, ${ }^{*}{ }^{3} \mathrm{M}$ C Falcão. ${ }^{1}$ GEPECIN - Science of Nutrition Group Research, Maringá, Paraná, Brazil; ${ }^{2}$ GEPEMENE - Nutrition, Exercise and Metabolism Group Research, Londrina, Paraná, Brazil; ${ }^{3}$ Children's Institute of School of Medicine of the University of São Paulo - Post-Graduate Program in Science, São Paulo, São Paulo, Brazil

Objective To analyse patterns of lifestyle is associated with abdominal obesity in Brazilian adolescents (14-18 years old).

Methods This school-based survey was carried out among high school students from Maringá/PR/Brazil, (2007) selected through two-stage random sampling. The sample included 991 (54.5\% girls) students. The outcome used was waist circumference (WC). The lifestyle variables used were: physical activity; sedentary behaviour and eating behaviour (number of meals per day; frequency of weekly consumption of soda). The multiple linear regression models were fitted to assess the relationship between WC and lifestyle patterns. Analyses were stratified by sex. Statistical significance was adopted $\alpha 5 \%$.

Results The mean waist circumference $(\mathrm{cm})$ for girls and boys was 77.3 and 80.4 , respectively, $(p<0.001)$. However, girls had higher prevalence of abdominal obesity than boys, $36.3 \%$ vs $28.4 \%$, respectively, $(p<0.001)$. The boys had a higher level of physical activity than girls, $436 \mathrm{~min} / \mathrm{wk}$ against $353 \mathrm{~min} / \mathrm{wk},(p=0.003)$, and the girls have higher sedentary behaviour, $7.1 \mathrm{~h} / \mathrm{d}$ compared to $5.9 \mathrm{~h} / \mathrm{d},(\mathrm{p}<0.001)$. The daily consumption of soda was positively associated with WC in both sexes, $B=0.56(p=0.041)$ for girls and $B=0.81$ ( $p=0.038$ ) for boys. And negatively associated between number of daily meals and WC, $\beta=-1.52(p<0.001)$ for girls and $\beta=-1.07(p=0.011)$ for boys.

Conclusion The higher prevalence of abdominal obesity, the lifestyle patterns are different between girls and boys and that eating habits are directly associated with waist circumference independent of sex.

\section{P2-59 ASSOCIATION OF BODY MASS INDEX AND FRUIT JUICE INTAKE IN 27 BRAZILIAN CITIES}

doi:10.1136/jech.2011.142976h.94

${ }^{1} \mathrm{~A}$ de Moura Souza, ${ }^{*}{ }^{1,2} \mathrm{I} N$ Bezerra, ${ }^{1} \mathrm{R}$ Sichieri. ${ }^{1}$ Department of Epidemiology, Institute of Social Medicine, State University of Rio de Janeiro, Rio de Janeiro, Rio de Janeiro, Brazil; ${ }^{2}$ Department of Clinical and Experimental Medicine, State University of Rio de Janeiro, Rio de Janeiro, Rio de Janeiro, Brazil

Introduction Fruit juice intake has been associated with weight gain in children and adolescents; however literature regarding this association in adults is scarce. 
Objective to investigate the association between body mass index (BMI) and fruit juice intake in Brazilian adults.

Method The Telephone Survey System for the Surveillance of Risk and Protective Factors for Chronic Non-Communicable Diseases (VIGITEL) is conducted every year since 2006. In 2008 was added a question regarding fruit juice intake. We analysed 2008 and 2009 data including 89.841 adults (20-65 years) from 27 Brazilian cities investigated. Weighted regression analyses included sex, age (years), schooling (years), level of physical activity (active/inactive) as independent variables, and BMI $\left(\mathrm{kg} / \mathrm{m}^{2}\right)$ as the dependent variable. Eating habits were evaluated based on the daily frequency of intake of fruit juices, fruits, vegetables and sugar-sweetened soft drinks.

Results Fifty-three percent of the participants were female, mean age was 37.2 years, mean BMI was $25 \mathrm{~kg} / \mathrm{m}^{2}$, and $19 \%$ reported a daily intake of fruit juice. BMI was negatively and significantly associated with fruit juice intake $(B=-0.29$; $p$-value $<0.0001$ for women; $B=-0.23$; $p$-value $=0.0004$ for men). There is a small negative association of fruit juice intake with soft drinks consumption and inactivity. After adjustment for sex, age, schooling, level of physical activity, intake of fruits, vegetables and soft drinks this association remained statistically significant $(~ B=-0.10$; $p$-value $=0.03$ ).

Conclusion Among Brazilian adults fruit juice intake may be a marker of healthier eating habits.

\section{P2-60 FREQUENCY OF OUT-OF-HOME EATING AND DIETARY HABITS IN THE BRAZILIAN TELEPHONE-BASED SURVEILLANCE SYSTEM}

doi:10.1136/jech.2011.142976h.95

${ }^{1}$ I N Bezerra, ${ }^{2} \mathrm{~A}$ de Moura Souza, ${ }^{*} \mathrm{C}$ A Monteiro, ${ }^{1,2} \mathrm{R}$ Sichieri. ${ }^{1}$ Department of Clinical and Experimental Medicine, University of the State of Rio de Janeiro, Rio de Janeiro, Rio de Janeiro, Brazil; ' $D$ Department of Epidemiology, Institute of Social Medicine, University of the State of Rio de Janeiro, Rio de Janeiro, Rio de Janeiro, Brazil; ' ${ }^{3}$ Department of Nutrition, School of Public Health, University of Sao Paulo, São Paulo, São Paulo, Brazil

Introduction The proportion of food purchased for out-of-home eating in Brazil has been increasing.

Objective To evaluate dietary habits associated with out-of-home eating among Brazilian adults.

Methods We analysed data of 135249 adults (20-65 y), living in 27 Brazilian cities, from the 2007, 2008 and 2009 Telephone Survey System for the Surveillance of Risk and Protective Factors for Chronic Non-Communicable Diseases (VIGITEL). Frequency of outof-home eating was based on the reported frequency of consuming lunch and dinner away from home per week. Dietary habits were based on the reported consumption of visible fat in meat and chicken and the daily consumption of soft drink, fruit and milk. Frequencies were weighted and analysis incorporated the complex design of the survey.

Results Men reported eating out more frequently than women (52.2\% vs $39.4 \%$ for at least once per week). Consumption of visible fat and soft drink increased with the frequency of out-of-home eating $(p<0.008)$, whereas daily fruit and milk consumption decreased $(p<0.01)$. After controlling for age and years of schooling, the OR of consuming fruit and milk decreased with the frequency of out-of-home eating ( $\mathrm{OR}=0.76$ (95\% CI 0.68 to 0.86$), \mathrm{OR}=0.79(95 \%$ CI 0.70 to 0.90 ) among men and $\mathrm{OR}=0.69$ (95\% CI 0.62 to 0.76 ), $\mathrm{OR}=0.76(95 \%$ CI 0.69 to 0.84$)$ among women, respectively, comparing 5 or more times/week with no out-of-home eating).

Conclusion Frequency of out-of-home eating was positively associated to negative markers of dietary habits and negatively associated to healthy markers of dietary habits among Brazilian adults. Due to the increasing out-of-home eating, public health strategies should be developed.

\section{P2-61 CHILDHO0D OBESITY AND PARENTAL SMOKING AS RISK FACTORS FOR CHILDHOOD ADHD IN LIVERPOOL CHILDREN}

doi:10.1136/jech.2011.142976h.96

${ }^{1} \mathrm{G}$ Koshy, ${ }^{2} \mathrm{~A}$ Delpisheh, ${ }^{* 1} \mathrm{~B}$ Brabin. ${ }^{1}$ Child and Reproductive Health Group, Liverpool School of Tropical Medicine, Liverpool, UK; ${ }^{2}$ Epidemiology Department, Ilam University of Medical Sciences, Ilam, Iran

Introduction $\mathrm{ADHD}$ prevalence has risen in parallel with rising prevalence of pregnancy smoking and childhood obesity. The objective was to determine the epidemiological association of pregnancy smoking and childhood obesity with ADHD.

Methods A cross-sectional community study was conducted in 2006 using a parental questionnaire. A total of 1074 schoolchildren aged 5-11 years were enrolled from 15 primary schools in a lower socioeconomic area of Merseyside. ADHD was defined by the question "does your child have Attention Deficit Hyperactivity Disorder, (ADHD), which has been diagnosed by a doctor?"

Results The prevalence estimates for childhood obesity, maternal smoking during pregnancy and childhood ADHD were 14.9\% (116/ 777), 28.0\% (269/955), and 3.4\% (32/945), respectively. ADHD prevalence was increased in children with obesity (RR $4.80,95 \% \mathrm{Cl}$ 2.2 to $10.4, \mathrm{p}<0.001$ ) and in children of mothers who smoked during pregnancy (RR $2.44,95 \%$ CI 1.2 to $4.9, \mathrm{p}=0.02$ ). Regression analysis adjusting for obesity, overweight, maternal smoking during pregnancy, heavy maternal smoking, household member smoking during pregnancy, doctor-diagnosed asthma, preterm birth, and low birthweight showed significant independent associations of ADHD prevalence with obesity (AOR 4.66, 95\% CI 1.57 to $13.89, p=0.006$ ) and pregnancy smoking (AOR 3.19, 95\% CI 1.08 to 9.49, $\mathrm{p}=0.04$ ). There was a positive dose-response association of $\mathrm{ADHD}$ with the number of maternal cigarettes smoked during pregnancy.

Conclusion Measures to reduce both smoking among pregnant women and childhood obesity might reduce prevalence of childhood $\mathrm{ADHD}$.

\section{P2-62 EARLY DETECTION OF COLORECTAL CANCER IN THE US IN APPALACHIA}

doi:10.1136/jech.2011.142976h.97

M Dignan, ${ }^{*}$ B Shelton, K Pearce, N Schoenberg, C Tolle, S Slone. University of Kentucky, Lexington, Kentucky, USA

Colorectal cancer is currently the $3^{\text {rd }}$ leading cause of cancer death worldwide. For rural, medically underserved populations in the US, and particularly in Appalachia, the burden of this disease is even greater. Elevated colorectal cancer rates in rural populations represents a missed opportunity for early detection through screening. Low screening rates are particularly concerning for Appalachia because of poverty and limited access to healthcare. To address this problem, a 5 -year study to increase screening through outreach to rural primary care providers was conducted from 2005 to 2010. Practices were randomised to early or delayed intervention groups and an intervention was provided in personal visits at the practices. The intervention was designed to assist practices in identifying opportunities to increase screening by providing education regarding screening efficacy, clinical performance measures, patient counselling, and creating a screening-friendly practice environment. Evaluation data were collected through medical record reviews before and after intervention delivery. Baseline data for both study groups indicated that healthcare providers had completed screening by any method in $36.7 \%$ of the 3906 patient records patient records reviewed. There was no significant difference in screening rates between the early or delayed group practices at baseline. At followup, screening rates remained low overall, $37.9 \%$. However, for practices that received the intervention, analyses of follow-up data 